\title{
Development of an enzyme linked immunosorbent assay for detection of cyathane diterpenoids
}

Tian Shen ${ }^{1}$, Lena M Hof ${ }^{1}$, Heike Hausmann², Marc Stadler ${ }^{3}$ and Holger Zorn ${ }^{1 *}$

\begin{abstract}
Background: So-called cyathane type diterpenoids are produced as secondary metabolites by basidiomycetes. Based on their antibacterial, fungicidal, and cytotoxic properties, cyathane type terpenoids represent interesting target compounds in fungal biotechnology.

Results: An indirect competitive enzyme linked immunosorbent assay has been developed for detection of cyathane type diterpenoids. Rabbit polyclonal antibodies were raised against a mixture of striatal A and B conjugated to bovine serum albumin. The conditions for direct attachment of the hapten striatal $B$ to a solid phase by passive adsorption were optimized. The cross reactivities of the striatals $A, C$ and $D$, of the striatins $A$ and $B$, and of the erinacines $C$ and $P$ to striatal $B$ were determined. The validation study showed that the ELISA was precise and sensitive. The average $I_{50}$ of striatal B was $36.0 \mathrm{ng} \mathrm{mL}^{-1}$ with an inter-assay coefficient of variation (CV) of 13.2\% ( $\left.n=5\right)$. Recoveries from striatal $B$ spiked samples in the assay were in the range of $97.3-125.9 \%$. A good correlation between the striatal $B$ concentration measured by the ELISA and by HPLC-DAD ( $\left.y=1.1122 x-0.1585, R^{2}=0.9942\right)$ was obtained from linear regression analysis. The suitability of the ELISA for detection of cyathane type diterpenoids in submerged cultures and fruiting bodies of $\mathrm{H}$. erinaceus was studied. It showed cross reactivity with supernatants from submerged cultures and extracts thereof, but did not show cross reactivity with extracts from fruiting bodies.

Conclusions: The developed method is appropriate for qualitative and quantitative detection of cyathane diterpenoids in complex mixtures. Due to its high sensitivity and specificity, it represents an ideal screening method for discovering new cyathane diterpenoids and new potential producers of them.
\end{abstract}

Keywords: Cyathane diterpenoids, ELISA, Hericium erinaceus, Striatal, Erinacine

\section{Background}

Cyathine and allocyathine were the first reported cyathane diterpenoids discovered by Allbutt and Ayer from a static liquid culture of the fungus Cyathus helenae (H. J. Brodie) in the early 1970 s $[1,2]$. They were proven to be active against actinomycetes, Gram-positive and Gram-negative bacteria, and some fungi, including dermatophytes. Afterwards, various structurally related compounds, so-called cyathane type diterpenoids, were isolated from different basidiomycetous cultures, e.g. striatals and striatins from Cyathus spp., sarcodonins from Sarcodon spp., and erinacines from Hericium spp. (Figure 1) [3-7]. All of these compounds share a cyathane skeleton consisting of five-, six-, and seven membered rings and possess antibacterial,

\footnotetext{
* Correspondence: holger.zorn@uni-giessen.de

1 Justus Liebig University Giessen, Institute of Food Chemistry and Food Biotechnology, Heinrich-Buff-Ring 58, Giessen 35392, Germany

Full list of author information is available at the end of the article
}

fungicidal, and cytotoxic properties. Fungal extracts prepared from $C$. striatus showed significant inhibitory effects on the NF-kB activation pathway and might be applied for cancer therapeutics [8]. Erinacines promote nerve growth factor (NGF) synthesis, which suggests the application of H. erinaceus or its secondary metabolites for the treatment and prevention of dementia and further neurodegenerative diseases [7,9-12].

Because of their interesting biological activities and their high potential in medicinal and pharmaceutical applications, cyathane diterpenoids have attracted increasing interest in recent years. At present, the most common method for analysis of cyathane diterpenoids is high performance liquid chromatography (HPLC) coupled to diode array and mass spectrometric detection [13].

As a rapid, sensitive and cost effective method, an ELISA specific for cyathane type secondary fungal metabolites may serve as a complementary method, especially in 


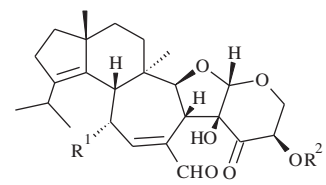

Striatal A: $\mathrm{R}^{1}=\mathrm{H}, \mathrm{R}^{2}=\mathrm{COCH}_{3}$ Striatal B: $\mathrm{R}^{1}=\mathrm{OH}, \mathrm{R}^{2}=\mathrm{COCH}_{3}$ Striatal C: $\mathrm{R}^{1}=\mathrm{OH}, \mathrm{R}^{2}=\mathrm{H}$ Striatal D: $\mathrm{R}^{1}=\mathrm{R}^{2}=\mathrm{H}$

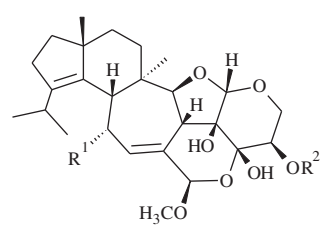

Striatin A: $\mathrm{R}^{1}=\mathrm{H}, \mathrm{R}^{2}=\mathrm{COCH}_{3}$ Striatin $\mathrm{B}: \mathrm{R}^{1}=\mathrm{OH}, \mathrm{R}^{2}=\mathrm{COCH}_{3}$ Striatin $\mathrm{C}: \mathrm{R}^{1}=\mathrm{OH}, \mathrm{R}^{2}=\mathrm{H}$

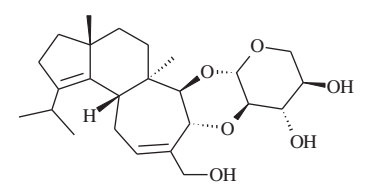

Erinacine C

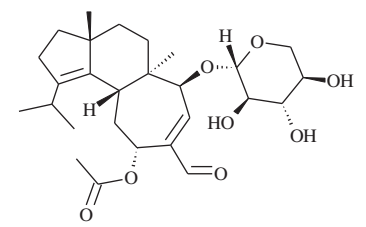

Erinacine $\mathrm{P}$

Figure 1 Structures of striatals, striatins and erinacines.

screenings for new producer strains. In the present study, an indirect competitive ELISA for the structure specific detection of cyathane type diterpenoids using polyclonal antibodies was developed, and its potential for the analysis of biological samples was proven.

\section{Results}

\section{Optimum coating conditions}

Striatal B was chosen as coating hapten in ELISA, because it is more polar than striatal A. In order to increase its solubility in aqueous solution, an equal volume of DMSO was added to the buffers. The optimum coating conditions (best signal-to-noise ratio and concentration dependence) were obtained by coating the plate in PBS/DMSO (1/1, $\mathrm{v} / \mathrm{v})$ buffer. More intense signals were obtained by coating at $24^{\circ} \mathrm{C}$ or $37^{\circ} \mathrm{C}$, compared to coating at $4^{\circ} \mathrm{C}$. Striatal B was adsorbed more efficiently by coating overnight than for $2 \mathrm{~h}$. Based on these results, the optimum coating conditions for striatal $\mathrm{B}$ are summarized as follows: PBS/DMSO (1/1, v/v) buffer, and coating overnight at $24^{\circ} \mathrm{C}$. The optimum concentration for coating of the hapten and the dilution of polyclonal antibodies (pAbs) were determined by checkerboard titration to be 5 $\mu \mathrm{g} \mathrm{mL} \mathrm{m}^{-1}$ and 1:200, respectively, which were used in the following indirect competitive ELISA experiments.

\section{Tolerance against organic solvents}

Due to the poor solubility of cyathane diterpenoids in aqueous solutions, the tolerance against organic solvents used to dissolve these compounds was tested for assay optimization. An addition of 5\% DMSO, acetonitrile or methanol, respectively, to the pre-incubation solution was found acceptable. Methanol could be used up to $25 \%$ without a notable negative effect. High percentages of DMSO up to $30 \%$ and of acetonitrile up to $20 \%$ resulted in considerable negative effects on the assay. An addition of DMSO $>30 \%$ or acetonitrile $>20 \%$ showed a sudden increase of signals. Since methanol reacts with striatals [4], an addition of 5\% DMSO or acetonitrile was selected for preincubation in the following indirect competitive ELISA.

\section{Cross reactivity}

Figure 2 illustrates a typical standard calibration curve of striatal B. The specificity of the pAbs against striatal B was evaluated by determination of the cross reactivities of several structurally related compounds, such as striatals $\mathrm{A}, \mathrm{C}$, and $\mathrm{D}$, striatins $\mathrm{A}$ and $\mathrm{B}$, and erinacines $\mathrm{C}$ and $\mathrm{P}$. The $\mathrm{IC}_{50}$ values are given in Table 1 . All of the tested cyathane diterpenoids showed cross reactivities against striatal B. No color reaction was detected with noncoated plates as blanks.

\section{Assay precision}

Intra-assay and inter-assay precisions were determined for several concentrations of striatal B (Table 2). The intra-assay precision was calculated from the extinction difference at $450 \mathrm{~nm}$ and $630 \mathrm{~nm}$ within one microtiter plate. Less than $10 \% \mathrm{CV}$ were obtained in every experiment, indicating an acceptable precision. The inter-assay precision was obtained from $\% \mathrm{~B} / \mathrm{B}_{0}$-values of 8 plates on different days. Variations of less than $20 \%$ were observed. The average $\mathrm{IC}_{50}$ of striatal B standard was $36.0 \mathrm{ng} \mathrm{mL}-1$ with an inter-assay CV of $13.2 \%(n=5)$.

\section{Recovery}

To assess the assay accuracy, striatal B was added to the sample matrix in several concentrations, and the recoveries of the spiked samples were determined. In parallel, HPLC-DAD was used to analyze the spiked samples. The apparent recoveries of striatal $B$ in the sample matrix as determined by indirect competitive ELISA ranged from $97.3 \%$ to $125.9 \%$, while those obtained by HPLC-DAD varied from $95.8 \%-110.2 \%$. The limit of quantification was $0.02 \mu \mathrm{g} \mathrm{mL} \mathrm{m}^{-1}$ for ELISA, and $0.40 \mu \mathrm{g}$ 

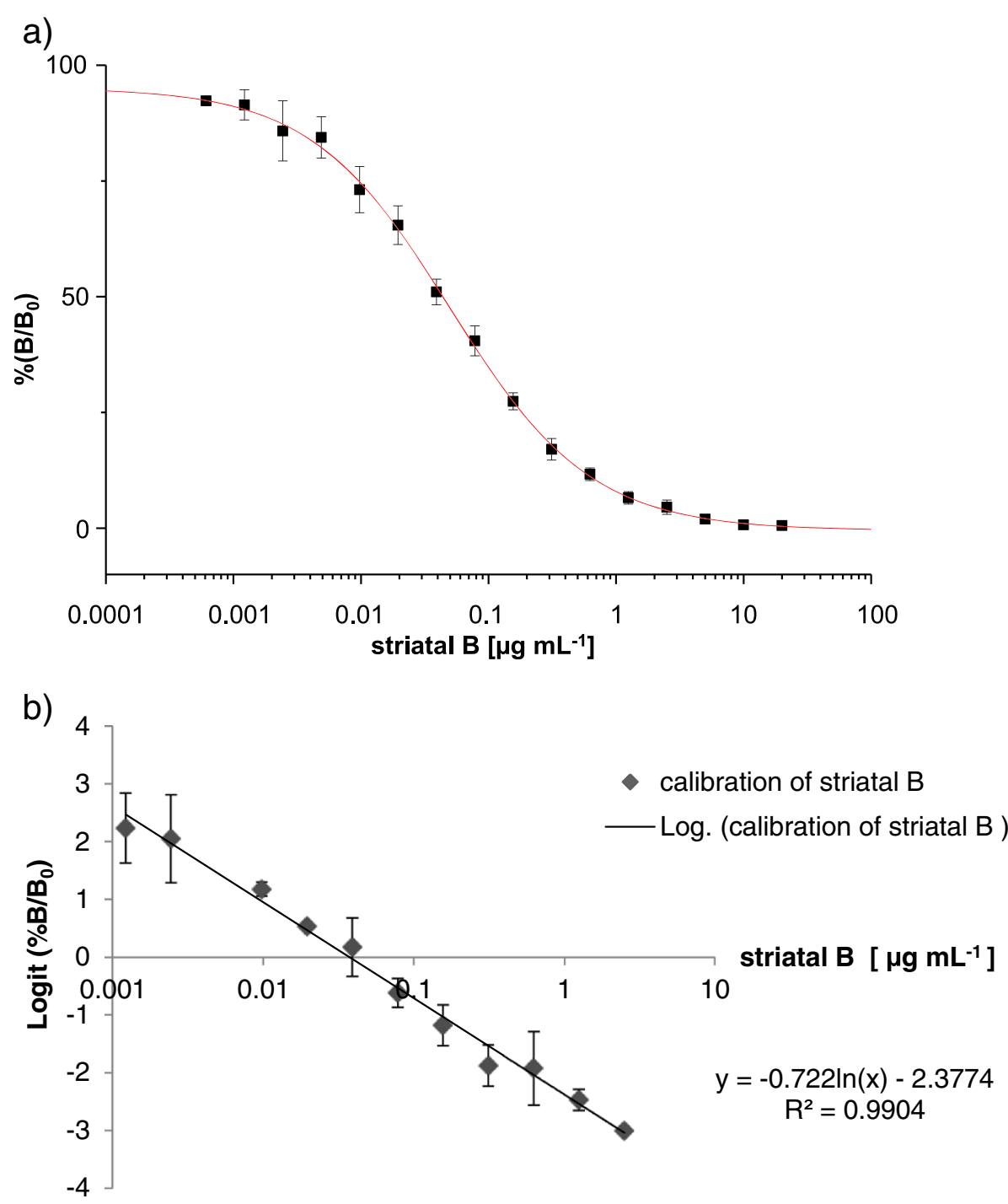

Figure 2 Calibration graphs for the quantification of striatal B. (a) Example of sigmoidal calibration curve for quantification of striatal B by indirect competitive ELISA. (b) Example of linearity of striatal B calibration curve. The linear working range was found to be $0.001-2.5 \mu \mathrm{g} \mathrm{mL}$, $y=-0.722 \ln (x)-2.3774, R^{2}=0.9904$

Table 1 Cross reactivities of structurally related compounds to striatal B

\begin{tabular}{ll}
\hline Compounds & Cross reactivity [\%]* \\
\hline Striatal B & 100.0 \\
Striatal A & 39.8 \\
Striatal C & 77.8 \\
Striatal D & 74.4 \\
Striatin A & 24.1 \\
Striatin B & 109.3 \\
Erinacine C & 60.0 \\
Erinacine P & 21.1 \\
\hline
\end{tabular}

*Related to molecular ratio.

Table 2 Intra- and inter-assay precision

\begin{tabular}{lll}
\hline $\begin{array}{l}\text { Concentration } \\
{\left[\boldsymbol{\mu g} \mathbf{~ m}^{-1}\right]}\end{array}$ & $\begin{array}{l}\text { Intra-assay }(\mathbf{n}=\mathbf{3}) \\
\mathbf{C V}[\%]\end{array}$ & $\begin{array}{l}\text { Inter-assay }(\mathbf{n}=\mathbf{8}) \\
\mathbf{C V}[\%]\end{array}$ \\
\hline 0.63 & 2.7 & 16.3 \\
0.32 & 3.9 & 14.8 \\
0.16 & 1.7 & 16.3 \\
0.08 & 2.4 & 8.3 \\
0.04 & 4.5 & 10.4 \\
0.0006 & 5.1 & 3.6 \\
\hline
\end{tabular}




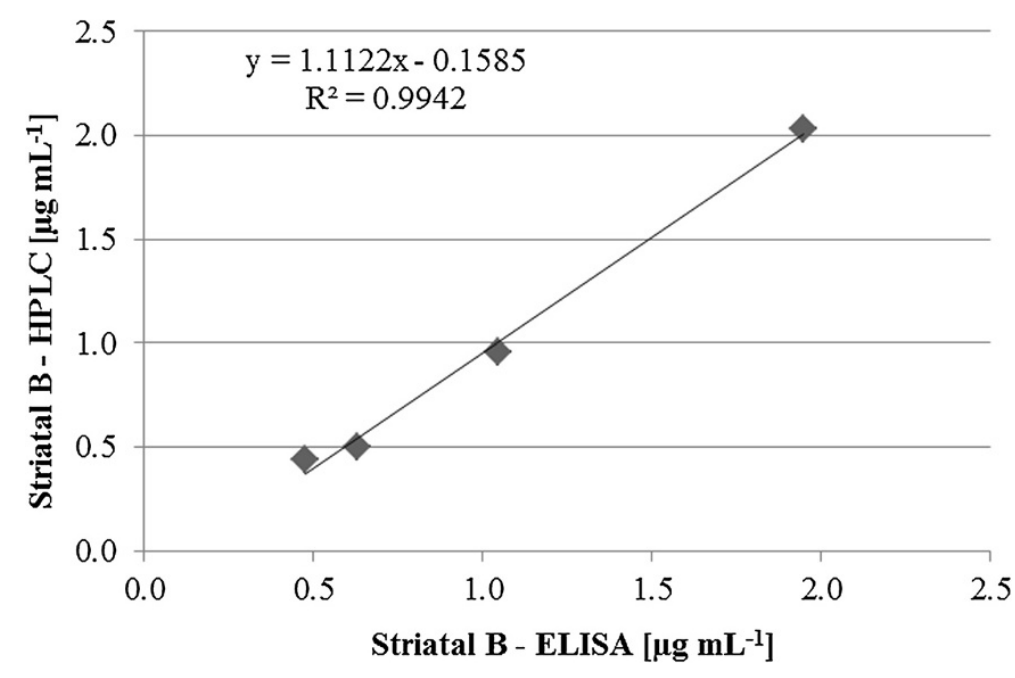

Figure 3 Correlation between striatal B concentrations in spiked samples measured by ELISA ( $x$-axis) and by HPLC-DAD (y-axis).

$\mathrm{mL}^{-1}$ for HPLC-DAD. A good correlation between the striatal $\mathrm{B}$ concentrations measured by ELISA and by HPLC-DAD ( $\left.\mathrm{y}=1.1122 \times-0.1585, \mathrm{R}^{2}=0.9942\right)$ was observed (Figure 3, Table 3).

\section{Analysis of $H$. erinaceus}

The indirect competitive ELISA was applied to samples prepared from submerged cultures as well as from fruiting bodies of $H$. erinaceus. The total concentrations of cyathane diterpenoids in supernatants and in extracts thereof are displayed in Figure 4. A similar change of \% $\mathrm{B} / \mathrm{B}_{0}$-values was observed in both cases during the 8 culture days. $\% \mathrm{~B} / \mathrm{B}_{0}$-values in extracts of fruiting bodies from $H$. erinaceus were near to $100 \%$ and were independent from the sample concentration in the assay.

\section{Discussion}

In the present study, striatal B was directly attached as a low molecular weight hapten to microtiter plates. In many studies the use of protein conjugated haptens for immobilization was reported [14-16], as the ability of proteins to adsorb to the plastic surface of microtiter plates is much higher than that of low molecular weight haptens. However, the coupling ratio of haptens to carrier proteins may vary depending on the coupling reaction and a waste of valuable haptens during conjugate synthesis is often unavoidable. The involvement of carrier proteins results in several problems. Firstly, it may cause unspecific binding to pAbs and thus reduce specific signals. Secondly, due to the large molecular dimension of carrier proteins, a shielding or masking effect may occur that impedes the binding of pAbs to the conjugated haptens [17]. Some researchers reported about pre-treatments of the plate surface, e.g. by UV irradiation or reagents in order to realize a direct binding of small molecules [18-20]. In the present study, the target analyte (molecular weight $<500 \mathrm{~g} \mathrm{~mol}^{-1}$ ) was directly attached to microtiter plates without pre-

Table 3 Recovery of spiked striatal $B$ in sample matrix

\begin{tabular}{|c|c|c|c|c|}
\hline \multirow{2}{*}{$\begin{array}{l}\text { Spiked concentration } \\
{\left[\mu \mathrm{g} \mathrm{mL}^{-1}\right]}\end{array}$} & \multicolumn{2}{|l|}{ ELISA $(n=3)$} & \multicolumn{2}{|l|}{ HPLC-DAD $(n=2)$} \\
\hline & Detected $\left[\mu \mathrm{g} \mathrm{mL}^{-1}\right]$ & Recovery [\%] & Detected $\left[\mu \mathrm{g} \mathrm{mL}^{-1}\right]$ & Recovery [\%] \\
\hline 10.010 & out of calibration & - & $10.50 \pm 0.05$ & $104.8 \%$ \\
\hline 5.005 & out of calibration & - & $5.26 \pm 0.10$ & $105.1 \%$ \\
\hline 2.002 & $1.949 \pm 0.219$ & $97.3 \%$ & $2.03 \pm 0.08$ & $101.5 \%$ \\
\hline 1.001 & $1.048 \pm 0.199$ & $104.7 \%$ & $0.96 \pm 0.02$ & $95.8 \%$ \\
\hline 0.501 & $0.630 \pm 0.291$ & $125.9 \%$ & $0.50 \pm 0.00$ & $99.6 \%$ \\
\hline 0.400 & $0.478 \pm 0.062$ & $119.3 \%$ & $0.44 \pm 0.02$ & $110.2 \%$ \\
\hline 0.200 & $0.252 \pm 0.026$ & $113.0 \%$ & n.d. & - \\
\hline 0.020 & $0.021 \pm 0.001$ & $102.8 \%$ & n.d. & - \\
\hline 0.001 & n.d* & - & n.d. & - \\
\hline
\end{tabular}

*n.d: not detected. 


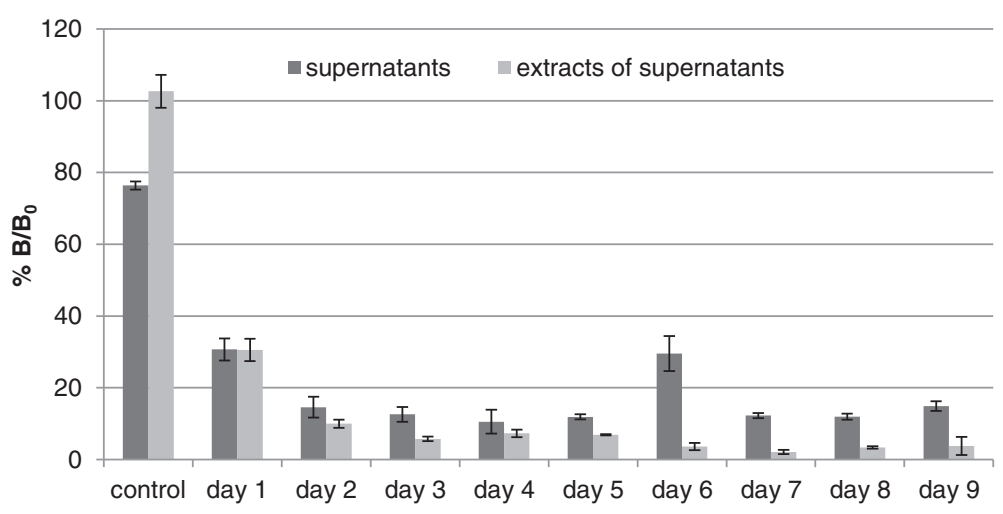

Figure 4 Analysis of supernatants of submerged cultures of $H$. erinaceus and extracts thereof during 8 culture days. Control: culture medium without $H$. erinaceus.

treatment of the plate surface. Significant differences between the various coating procedures were found during the study of coating conditions. The $\mathrm{pH}$ of the coating buffer significantly affected the coating efficiency. The final $\mathrm{pH}$ of the coating buffer PBS, potassium acetate and sodium carbonate after mixing with DMSO was 9.7, 5.8 and 11.1, respectively. An influence of the $\mathrm{pH}$ on the coating efficiency has been reported in several publications [21-23]. In addition, a decomposition of striatal B was detected in potassium acetate buffer in a time course study by HPLC analysis (data not shown). That might be a reason for the poor attachment of striatal $\mathrm{B}$ to the plate in this buffer system. For striatal $\mathrm{B}$, coating at $\mathrm{pH}$ 9.7 was more efficient than at $\mathrm{pH}$ 11.1.

The presence of organic solvents may influence the performance of an ELISA. Methanol caused the least negative effect, and the use of low concentrations of acetonitrile or DMSO was also acceptable. Similar results have been reported e.g. by $[17,23,24]$. The tolerance against organic solvents allows for the analysis of samples from fungal cultures which require extraction with an organic solvent. The sudden increase of the signals in the presence of acetonitrile and DMSO in high concentrations indicated a false positive signal possibly caused by denaturation of the antibodies and thus an unspecific adsorption to the blocking proteins.

All of the examined cyathane diterpenoids showed cross reactivities against striatal B. As expected, striatin B with $\mathrm{IC}_{50}=109.3 \%$ showed the highest cross reactivity. Striatin $B$ and striatal B differ only in the aldehyde group at the $\mathrm{C}_{7}$ ring, and this position of striatal $\mathrm{B}$ was used for BSA-conjugate synthesis for immunization. Therefore, striatin B and striatal B possess the same antigenic determinants. Erinacine $P$ showed the lowest cross reactivity with $\mathrm{IC}_{50}=21.1 \%$. Based on the cross reactivities and molecular structures it might be suspected that the resulting antiserum displays the highest affinity toward the cyathane ring moiety.
The recovery experiments suggested that the developed ELISA is accurate and significantly more sensitive than the HPLC-DAD method. It could be used for the estimation of the total cyathane diterpenoid concentrations of biological samples. The assay showed interferences with constituents of the culture media of $\mathrm{H}$. erinaceus, as a matrix effect was observed with the control samples (cf. Figure 4, control). Therefore, the use of organic extracts is preferred for the analysis of cyathane type diterpenoids in fungal culture supernatants. The product concentrations could be estimated during the cultivation period. However, the total concentrations represent the sum of all compounds showing cross reactivity against striatal B. They may thus differ from the individual concentrations determined by HPLC.

No significant interferences $\left(\% \mathrm{~B} / \mathrm{B}_{0}\right.$-values close to $\left.100 \%\right)$ were observed with crude extracts of fruiting bodies of $H$. erinaceus. Therefore, the secondary metabolites present in the fruiting bodies, e.g. hericenone and erinacerine, did not cross react with the polyclonal antibodies. These results are in good agreement with a review article about secondary metabolites from $H$. erinaceus [12]. Up to date, erinacines have been mostly reported from submerged cultures of $H$. erinaceus, and only traces of erinacines have been found in fruiting bodies [25]. In the present study, the trace concentrations of erinacines in the fruiting bodies were apparently below the detection limit of the ELISA.

The CV\% values for the inter-assay precision might be caused by many factors, e.g. the position of the wells in the plate (edge effect), the day to day variation in reagent preparation, the temperature of buffers, the variability in washing procedures, pipetting errors and the loss of enzyme activity of the secondary antibodies after prolonged storage $[16,26]$.

\section{Conclusions}

Polyclonal antibodies against striatal A/B were produced, and an indirect competitive ELISA was developed for 
determination of striatal B and structural related compounds. The method has shown satisfactory results concerning specificity, sensitivity and accuracy. The application to samples from submerged cultures and fruiting bodies of $H$. erinaceus indicated that the ELISA may be used for the estimation of total cyathane diterpenoid concentrations in complex mixtures. Due to its high sensitivity and specificity, it represents an ideal screening tool for discovering new potential producers of cyathane type diterpenoids. Further optimization will be needed to improve the inter-assay precision.

\section{Methods}

\section{Chemicals and solutions}

All reagents were of analytical grade unless specified otherwise. Ultra-pure water (produced by arium ${ }^{\circ} 611 \mathrm{VF}$ Water System, Sartorius, Göttingen, Germany) was used for all solutions, media, and HPLC eluents.

Acetonitrile (HPLC gradient grade), calcium carbonate, Edamin ${ }^{\circ} \mathrm{K}, 30 \%$ hydrogen peroxide $\left(\mathrm{H}_{2} \mathrm{O}_{2}\right)$, and D-mannitol were purchased from Sigma Aldrich (Steinheim, Germany).

Salts, Agar-Agar, bovine serum albumin (BSA), EDTA, $\alpha$ - $\mathrm{D}(+)$-glucose monohydrate, soy peptone, sucrose, 3,3,5,5'-tetramethyl benzidine (TMB), Tween ${ }^{\circ} 20$, yeast extract, and organic solvents were purchased from Applichem (Darmstadt, Germany) or from Roth (Karlsruhe, Germany).

Malt extract was obtained from Fluka, Neu-Ulm, Germany. Molasses was obtained from Südzucker, Offstein, Germany. Oatmeal was provided from Dr. Oetker, Düsseldorf, Germany.

Standards of erinacines and striatals, originally isolated from the two selected producer strains, were obtained from the library of pure natural products of InterMed Discovery, Dortmund, Germany (IMD, formerly Bayer Healthcare) and their purity and identity was confirmed by 2D-NMR and HR-MS, prior to their use in the experiments. Additionally, striatals A and B (for immunization) and striatals $\mathrm{C}$ and $\mathrm{D}$, striatins $\mathrm{A}$ and $\mathrm{B}$, and erinacines $\mathrm{C}$ and $\mathrm{P}$ (for determination of cross reactivities) were extracted and purified from submerged cultures of the basidiomycetes Cyathus striatus and Hericium erinaceus. The structures were confirmed by comparison to the pure reference compounds and by NMR analysis (cf. Additional file 1: Supplementary material).

Solutions for ELISA:

Coating buffers:

1. $10 \mathrm{mM}$ phosphate buffered saline (PBS), $\mathrm{pH} 7.4$ mixed with DMSO, $1 / 1$, v/v.

2. $0.1 \mathrm{M}$ potassium acetate buffer, $\mathrm{pH} 4.0$ mixed with DMSO, $1 / 1, \mathrm{v} / \mathrm{v}$.
3. $0.1 \mathrm{M}$ sodium carbonate buffer, $\mathrm{pH} 9.6$ mixed with DMSO, $1 / 1, \mathrm{v} / \mathrm{v}$.

Washing buffer: $10 \mathrm{mM}$ PBS, pH 7.4 with $0.05 \%$ Tween 20 (PBST).

Blocking reagent (1\% gelatin) was purchased from Roche Diagnostics (Mannheim, Germany). Secondary antibodies (goat anti-rabbit $\lg$ peroxidase conjugate) were obtained from Merck (Darmstadt, Germany).

Color development: $0.12 \mathrm{mg} \mathrm{mL}{ }^{-1} \mathrm{TMB}$ and $0.05 \%$ $\mathrm{H}_{2} \mathrm{O}_{2}$ in $0.05 \mathrm{M}$ sodium acetate solution, pH 4.5 (TMB solution). This solution was freshly prepared before use.

Stopping reagent: $0.5 \mathrm{M}$ sulfuric acid.

\section{Fungal strains}

Cyathus striatus (STMA07048, isolated from basidiospores of a specimen from a trunk of Picea collected in July 1997 in Stelzenberg, Rheinland-Pfalz, Germany) and Hericium erinaceus (FU70034, isolated from basidiocarp tissue) for submerged culture were obtained from IMD. Prior to the experiments, the identity of both strains was confirmed by microscopic studies and by comparison of their ITS nrDNA sequences with reference data in Genbank. The fungi were maintained on a solid medium containing 20 $\mathrm{g} \mathrm{L}^{-1}$ malt extract and $15 \mathrm{~g} \mathrm{~L}^{-1}$ Agar-Agar.They are deposited in the culture collection of IMD Natural Solutions $\mathrm{GbR}$ (formerly InterMed Discovery $\mathrm{GmbH}$ under liquid nitrogen. A duplicate strain and the corresponding specimen of the C. striatus material is also maintained at the personal herbarium and culture collection of Marc Stadler.

Fruiting bodies of Hericium erinaceus were obtained from a commercial provider www.pilzgarten.de, Helvesiek, Germany.

\section{Culture media}

Soy peptone, yeast malt, and sugar molasses media were prepared according to [25].

\section{Instruments}

\section{HPLC-DAD}

The HPLC system was from Merck Hitachi, Darmstadt, Germany, and comprised a pump (L-7100), an auto sampler (L-7200), an interface (D-7000), and a diode array detector (L-7455, $200-600 \mathrm{~nm}$ ). Column: reversed phase, Nucleosil ${ }^{\circ} 100-5 \mathrm{C}_{18}$, CC $125 / 3 \mathrm{~mm}$ with a guard column Nucleosil ${ }^{\circ} 100-5 C_{18}$, CC 8/3 mm (Macherey Nagel, Düren, Germany).

Flow rate: $0.4 \mathrm{~mL} \mathrm{~min}^{-1}$ for striatal and striatin analysis and $0.6 \mathrm{~mL} \mathrm{~min}^{-1}$ for erinacine analysis.

Eluent: Acetonitrile (A) and ultra-pure water (B).

Wavelength: 210, 233 and $254 \mathrm{~nm}$.

Gradient for striatal and striatin: 50\% A (0 min) - 94\% A (14 min) - 94\% A (17 min) - 100\% A (19 min) - 100\% A $(25 \mathrm{~min})-50 \% \mathrm{~A}(30 \mathrm{~min})-50 \% \mathrm{~A}(40 \mathrm{~min})$. 
Gradient for erinacine: 30\% A (0 min) - 50\% A (15 min $)-50 \%$ A (16 min $)-100 \%$ A (23 min $)-100 \%$ A (38 min) $-30 \%$ A (43 min) - 30\% A (47 min).

\section{Preparative HPLC}

A semi-preparative HPLC system (Young Lin, Hongye Anyang, Korea) equipped with quaternary pump (YL9110S) and dual wavelength UV/Vis detector (YL 9120S), combined with a fraction collector (CHF 122SC, Advantec, Osaka, Japan) was used. A column Kromasil $100 \mathrm{C}_{18}, 7 \mu \mathrm{m}$, $250 \times 20 \mathrm{~mm}$ (MZ Analysentechnik, Mainz) with a guard column Kromasil $100 \mathrm{C}_{18}, 7 \mu \mathrm{m}, 50 \times 20$ mm (MZ) was used to isolate the target compounds.

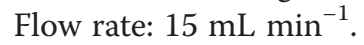

Eluent: Acetonitrile (A) and ultra-pure water (B).

Gradient for striatals and striatins: 50\% A (0 min) $50 \%$ A (10 min $)-80 \%$ A (55 min $)-80 \%$ A (70 min $)-$ $100 \%$ A ( $80 \mathrm{~min})-100 \%$ A (95 min). Wavelengths: 210 and $254 \mathrm{~nm}$.

Gradient for erinacines: $25 \mathrm{~A} \%(0 \mathrm{~min})-25 \% \mathrm{~A}(10$ min) $-48 \%$ A (35 min) - 48\% A (45 min) - 55\% A (52 $\min )-55 \%$ A $(60 \mathrm{~min})-100 \% \mathrm{~A}(90 \mathrm{~min})-100 \% \mathrm{~A}$ (110 min). Wavelengths: 210 and $233 \mathrm{~nm}$.

\section{ELISA}

The ELISA was performed in microtiter plates Immuno Plate Maxisorp F96 (Nunc, Denmark). Incubation steps and color development were performed in an incubator INE 500 (Memmert, Schwabach, Germany). Washing was done by an eight-channel pipette Research Pro 50-1200 $\mu \mathrm{L}$ (Eppendorf, Wesseling-Berzdorf, Germany). The extinction was measured by a microtiter plate reader Synergy 2 (BioTek, Bad Friedrichshall, Germany).

\section{Biosynthesis and isolation of cyathane diterpenoids Submerged cultivation}

Pre-cultures of $C$. striatus were grown submerged in $250 \mathrm{~mL}$ Erlenmeyer flasks containing $100 \mathrm{~mL}$ soy peptone medium at $24^{\circ} \mathrm{C}$ and $150 \mathrm{rpm}$ for 7 days. After that, the mycelium was homogenized with an UltraTurrax (IKA, Staufen, Germany) at 10,000 rpm for 10 sec. $40 \mathrm{~mL}$ homogenized mycelium were inoculated into a new 1,000 mL Erlenmeyer flask containing 400 $\mathrm{mL}$ soy peptone medium and incubated at $24^{\circ} \mathrm{C}$ and $150 \mathrm{rpm}$ for further 12 days.

Pre-cultures of $H$. erinaceus were grown submerged in $250 \mathrm{~mL}$ Erlenmeyer flasks containing $100 \mathrm{~mL}$ yeast malt medium at $24^{\circ} \mathrm{C}$ and $150 \mathrm{rpm}$ for 7 days. Afterwards, $40 \mathrm{~mL}$ homogenized mycelium were inoculated into $400 \mathrm{~mL}$ sugar molasses medium in $1,000 \mathrm{~mL}$ Erlenmeyer flasks and incubated at $24^{\circ} \mathrm{C}$ and $150 \mathrm{rpm}$ for further 4 days to obtain erinacine $\mathrm{P}$ or 12 days to obtain erinacine $\mathrm{C}$.

\section{Extraction and isolation}

After submerged cultivation, the mycelia from $2.2 \mathrm{~L}$ culture media were separated from the supernatants by centrifugation at 2,880 $\mathrm{g}$ and $4^{\circ} \mathrm{C}$ for $10 \mathrm{~min}$ (Allegra ${ }^{\circ} \mathrm{X}-15 \mathrm{R}$, BECKMAN COULTER ${ }^{\mathrm{m}}$, Krefeld, Germany), and extracted twice with $400 \mathrm{~mL}$ ethyl acetate. The combined organic extracts were dried over sodium sulfate, and afterwards evaporated to dryness. A brown residue was obtained after evaporation, which was dissolved in acetonitrile and subjected to preparative HPLC. The extracts of Cyathus striatus contained the striatals A, B, C and $\mathrm{D}$. The striatins $\mathrm{A}$ and $\mathrm{B}$ were prepared by conversion of the striatals A and B by stirring in methanol at room temperature overnight, respectively. The erinacines $\mathrm{P}$ and $\mathrm{C}$ were isolated from the extracts of Hericium erinaceus.

\section{Production of polyclonal antibodies}

The aldehyde function of the striatals and $\mathrm{NH}_{2}$ functions of BSA were used for synthesis of striatal A/B (1/1, w/ w)-BSA conjugates. The ratio of striatals to BSA was determined by MALDI-TOF-MS. About 17 striatal molecules were coupled to one BSA molecule (Eurogentec S. A, Seraing, Belgien). The production of rabbit pAbs against striatal A/B was performed by Eurogentec S.A. Two rabbits with ID SA6928 and SA6929 were immunized four times with $200 \mu \mathrm{g}$ of the striatal A/B-BSA conjugate on day $0,14,28$ and 56. An additional immunization was done on day 91 with $400 \mu \mathrm{g}$ striatal A/B-BSA conjugate. The final antisera were collected on day 115 and stored at $-20^{\circ} \mathrm{C}$ without further purification until use. The pre-bleed sera of these two rabbits on day 0 before immunization were used as negative control in ELISA. The final antiserum from rabbit SA6928 was used in the following assays because of its higher specificity against striatal B compared to SA6929 (data not shown).

\section{Development of an indirect competitive ELISA Optimization of coating conditions}

Striatal B was directly attached to the plate in several different concentrations. The optimal conditions (buffer, temperature and time) for direct coating of striatal B were studied by an indirect non-competitive ELISA. The incubation temperatures were $4^{\circ} \mathrm{C}, 24^{\circ} \mathrm{C}$ and $37^{\circ} \mathrm{C}$, and the coating time was $2 \mathrm{~h}$ or overnight.

\section{Checkerboard titration}

The optimal dilution of pAbs and the optimal coating amount of striatal B were determined simultaneously by checkerboard titration (a two-dimensional titration method) by indirect non-competitive ELISA. Striatal B with different concentrations $\left(0.04\right.$ to $20 \mu \mathrm{g} \mathrm{mL}^{-1}$, two fold dilution) was coated to the microtiter plate and then bound to pAbs with serial dilutions (1:50 to 1:6,400, stepwise in two fold dilution). 


\section{Tolerance against organic solvents}

The tolerance against organic solvents (DMSO, acetonitrile, methanol) used to dissolve the cyathane diterpenoids was examined by indirect competitive ELISA. Different proportions of organic solvent $(5-50 \%, \mathrm{v} / \mathrm{v}, 0 \%$ as blank) were added to PBST with a final dilution of pAbs 1:200 and pre-incubated without competing molecules at $24^{\circ} \mathrm{C}$ for $1 \mathrm{~h}$. The effects of organic solvents on the ELISA system were evaluated by comparing the measured extinctions to those of the blanks.

\section{ELISA}

\section{Coating}

$100 \mu \mathrm{L}$ of $5 \mu \mathrm{g} \mathrm{mL} \mathrm{m}^{-1}$ striatal B in coating buffer 1 were pipetted in wells of a microtiter plate. $100 \mu \mathrm{L}$ of $1 \mu \mathrm{g} \mathrm{mL} \mathrm{L}^{-1}$ BSA diluted in PBS were pipetted in two wells as positive control. The incubation was performed overnight at $24^{\circ} \mathrm{C}$.

\section{Blocking}

$200 \mu \mathrm{L}$ of $1 \%$ gelatin were added to the wells and incubated at $24^{\circ} \mathrm{C}$ for $3 \mathrm{~h}$.

\section{Pre-incubation}

Serial dilutions of analyte were prepared in a concentration range of $0.0003-20 \mu \mathrm{g} \mathrm{mL} L^{-1}$ in PBST containing $5 \%$ acetonitrile or DMSO and a final pAbs dilution of 1:200. The incubation was performed for $1 \mathrm{~h}$ at $24^{\circ} \mathrm{C}$ in $2 \mathrm{~mL}$ Eppendorf cups.

\section{Incubation with pAbs}

$100 \mu \mathrm{L}$ pre-incubated analyte were added to each well (in triplicate) and incubated for $1 \mathrm{~h}$ at $24^{\circ} \mathrm{C}$. For the indirect non-competitive ELISA, $100 \mu \mathrm{L}$ pAbs with a 1:100 dilution in PBST were used without competitors instead of the pre-incubated analyte.

\section{Incubation with secondary antibodies}

$100 \mu \mathrm{L}$ secondary antibodies with a 1:5,000 dilution in PBST were added to each well and incubated for $1 \mathrm{~h}$ at $24^{\circ} \mathrm{C}$.

\section{Color development}

$100 \mu \mathrm{L}$ of the TMB solution were added to each well and incubated for $15 \mathrm{~min}$ at $24^{\circ} \mathrm{C}$. Without washing step, $100 \mu \mathrm{L}$ of $0.5 \mathrm{M}$ sulfuric acid were added to each well to stop the color development.

\section{Measurement}

The extinctions were measured immediately by a microtiter plate reader at $450 \mathrm{~nm}$ and $630 \mathrm{~nm}$.

Unless otherwise specified, the wells were washed with $300 \mu \mathrm{L}$ washing buffer PBST three times after each incubation step. The indirect non-competitive ELISA involved all of the steps of the indirect competitive ELISA, except for the pre-incubation of pAbs with competitors.

\section{Data analysis}

Striatal B standards and samples were analyzed in triplicates. The differences between extinctions at $450 \mathrm{~nm}$ and $630 \mathrm{~nm}$ were used for calculation and plotting the sigmoidal curves. $\% \mathrm{~B} / \mathrm{B}_{0}$-values for calibration curves were calculated as follows:

$$
\% \frac{B}{B_{0}}=\frac{\Delta E-\Delta E_{\text {excess }}}{\Delta E_{0}-\Delta E_{\text {excess }}} \times 100
$$

$\Delta E$ : the extinction differences of samples at $450 \mathrm{~nm}$ and $630 \mathrm{~nm}$.

$\Delta E_{0}$ : the extinction differences of the upper asymptote of the sigmoidal curve between $450 \mathrm{~nm}$ and $630 \mathrm{~nm}$.

$\Delta E_{\text {excess: }}$ : the extinction differences of the lower asymptote of the sigmoidal curve between $450 \mathrm{~nm}$ and $630 \mathrm{~nm}$.

\section{Cross reactivity $\left(\mathrm{IC}_{50}\right)$}

The assay specificity was evaluated by obtaining sigmoidal curves for several structurally related compounds (striatals $\mathrm{A}, \mathrm{C}$ and $\mathrm{D}$, striatins $\mathrm{A}$ and $\mathrm{B}$, and erinacines $\mathrm{C}$ and $\mathrm{P}$ ) as competitors against the striatal $\mathrm{B}$ standard. The calibration curve of striatal B standard was measured individually for each plate. The $\mathrm{IC}_{50}$ were estimated by using a linearization of the calibration curves with a logit-transformation of $\% \mathrm{~B} / \mathrm{B}_{0}$-values:

$$
\operatorname{logit}\left(\% B / B_{0}\right)=\ln \left(\frac{\% B / B_{0}}{100-\% B / B_{0}}\right)
$$

Cross reactivity $\left(\mathrm{IC}_{50}\right)=\mathrm{A} / \mathrm{B} \times 100 \%$.

A: concentration of striatal $B$ standard at logit $\left(\% B / B_{0}\right)=0$. $\mathrm{B}$ : concentration of competitors at logit $\left(\% \mathrm{~B} / \mathrm{B}_{0}\right)=0$.

The linear regression of $\operatorname{logit}\left(\% \mathrm{~B} / \mathrm{B}_{0}\right)$ was used for determination of concentrations in unknown samples.

\section{Recovery}

Acetonitrile was spiked with striatal B at several concentrations $\left(0.001-10 \mu \mathrm{g} \mathrm{mL} \mathrm{m}^{-1}\right)$ and analyzed by indirect competitive ELISA as well as by HPLC-DAD. The concentrations of striatal $\mathrm{B}$ of spiked samples were calculated from linear regression of logit $\left(\% \mathrm{~B} / \mathrm{B}_{0}\right)$ in ELISA and linear calibration for striatal $B$ in HPLC-DAD. The recoveries of striatal B were obtained by:

$$
\text { recovery }[\%]=\frac{\text { detected concentration }}{\text { spiked concentration }} \times 100 \%
$$

\section{Analysis of $H$. erinaceus samples}

The supernatants and extracts of supernatants from submerged cultures, as well as extracts of fruiting bodies of H. erinaceus were analyzed by indirect competitive ELISA: 


\section{Sample preparation for pre-incubation of supernatants in ELISA}

$50 \mu \mathrm{L}$ of supernatants from submerged cultures (culture day 1 to day 8) were added to $950 \mu \mathrm{L}$ PBST with a final DMSO proportion of $5 \%$ and a final pAbs dilution of 1:200. Culture medium without $H$. erinaceus was used as negative control.

\section{Sample preparation for pre-incubation of extracts of supernatants}

$20 \mathrm{~mL}$ of supernatants from submerged cultures (culture day 1 to 8 ) were extracted once with $20 \mathrm{~mL}$ ethyl acetate. $15 \mathrm{~mL}$ of the ethyl acetate phase was evaporated to dryness, and the residues were redissolved in $1 \mathrm{~mL}$ acetonitrile for ELISA and HPLC-DAD analysis. $5 \mu \mathrm{L}$ of the extracts were added to $995 \mu \mathrm{L}$ PBST with a final acetonitrile proportion of $5 \%$ and a final pAbs dilution of 1:200. Extracts of culture media without $H$. erinaceus were used as negative controls.

\section{Sample preparation for pre-incubation of extracts of fruiting bodies}

$32.6 \mathrm{~g}$ fruiting bodies of $\mathrm{H}$. erinaceus were homogenized and then extracted with $200 \mathrm{~mL}$ ethyl acetate under stirring overnight. The ethyl acetate phase was dried over sodium sulfate and evaporated to dryness after filtration. $1 \mathrm{mg}$ crude extract was dissolved in $1 \mathrm{~mL}$ acetonitrile. Several concentration levels $(50.0,10.0,5.0,2.5,1.0$ and $0.5 \mu \mathrm{g} \mathrm{mL} \mathrm{m}^{-1}$ ) of the extracts were used in the preincubation solution. Acetonitrile was used as blank.

\section{Availability of supporting data}

The data sets supporting the results of this article are included within the article and its additional file.

\section{Additional file}

Additional file 1: Supplementary material.

\section{Competing interests}

The authors declare that they have no competing interests.

\section{Authors' contributions}

TS purified the cyathane type diterpenoids, carried out the ELISA and drafted the manuscript. LH was involved in the studies on the cross reactivities, and $\mathrm{HH}$ was responsible for NMR analyses and data interpretation. MS provided the fungal strains and contributed to the discussion. $\mathrm{HZ}$ participated in the design of the study and helped to draft the manuscript. All authors read and approved the final manuscript.

\section{Acknowledgement}

The authors thank the BMBF and CLIB 2021 for financial support of the project (FKZ 0315404 A). Part of the work was supported by the excellence initiative of the Hessian Ministry of Science and Art which encompasses a generous grant for the LOEWE research focus 'Integrative Fungal Research'.

\section{Author details}

1Justus Liebig University Giessen, Institute of Food Chemistry and Food Biotechnology, Heinrich-Buff-Ring 58, Giessen 35392, Germany. ${ }^{2} J u s t u s ~ L i e b i g$ University Giessen, Institute of Organic Chemistry, Heinrich-Buff-Ring 58, Giessen 35392, Germany. ${ }^{3}$ Department Microbial Drugs, Helmholtz Centre for Infection Research, Inhoffenstrasse 7, Braunschweig 38124, Germany.

Received: 2 June 2014 Accepted: 5 November 2014 10.

\section{References}

1. Allbutt AD, Ayer WA, Brodie HJ, Johri BN, Taube H: Cyathin, a new antibiotic complex produced by Cyathus helenae. Can J Microbiol 1971, 17:1401-1407

2. Ayer WA, Taube $\mathrm{H}$ : Metabolites of Cyathus helenae. cyathin $\mathrm{A}_{3}$ and allocyathin $B_{3}$, members of a new group of diterpenoids. Tetrahedron Lett 1972, 13:1917-1920.

3. Anke T, Oberwinkler F: The striatins-new antibiotics from the basidiomycete Cyathus striatus (Huds. ex Pers.) Willd. J Antibiot 1977, 30:221-225.

4. Anke T, Rabe U, Schu P, Eizenhöfer T, Schrage M, Steglich W: Studies on the biosynthesis of striatal-type diterpenoids and the biological activity of herical. Z Naturforsch C 2002, 57:263-271.

5. Shibata H, Tokunaga T, Karasawa D, Hirota A, Nakayama M, Nozaki H, Tada T: Isolation and characterization of new bitter diterpenoids from the fungus Sarcodon scabrosus. Agric Biol Chem 1989, 53:3373-3375.

6. Shibata H, Irie A, Morita Y: New antibacterial diterpenoids from the Sarcodon scabrosus fungus. Biosci Biotechnol Biochem 1998, 62:2450-2452.

7. Kawagishi $\mathrm{H}$, Zhuang $\mathrm{C}$ : Compounds for dementia from Hericium erinaceum. Drugs Future 2008, 33:149-155.

8. Petrova RD, Mahajna J, Reznick AZ, Wasser SP, Denchev CM, Nevo E: Fungal substances as modulators of NF-KB activation pathway. Mol Biol Rep 2007 34:145-154.

9. Kawagishi H, Shimada A, Hosokawa S, Mori H, Sakamoto H, Ishiguro $Y$, Sakemi S, Bordner J, Kojima N, Furukawa S: Erinacines E, F, and G, stimulators of nerve growth factor (NGF)-synthesis, from the mycelia of Hericium erinaceum. Tetrahedron Lett 1996, 37:7399-7402.

10. Kawagishi $H$, Shimada A, Shirai R, Okamoto K, Ojima F, Sakamoto H, Ishiguro $Y$, Furukawa S: Erinacines A, B and C, strong stimulators of nerve growth factor (NGF)-synthesis, from the mycelia of Hericium erinaceum. Tetrahedron Lett 1994, 35:1569-1572.

11. Kawagishi H, Simada A, Shizuki K, Ojima F, Mori H, Okamoto K, Sakamoto H, Furukawa S: Erinacine D, a stimulator of NGF-synthesis, from the mycelia of Hericium erinaceum. Heterocycllic Commun 1996, 2:51-54.

12. Ma B, Shen J, Yu H, Ruan Y, Wu T, Zhao X: Hericenones and erinacines: stimulators of nerve growth factor (NGF) biosynthesis in Hericium erinaceus. Mycology 2010, 1:92-98.

13. Krzyczkowski W, Malinowska E, Herold F: Erinacine A biosynthesis in submerged cultivation of Hericium erinaceum: quantification and improved cultivation. Eng Life Sci 2010, 10:446-457.

14. Jahn C, Zorn H, Petersen A, Schwack W: Structure-specific detection of plant cuticle bound residues of chlorothalonil by ELISA. Pestic Sci 1999, 55:1167-1176

15. Xu DM, Yu XY, Liu YQ, Feng JT, Pan LG, Liu XJ, He J, Zhang X: Development of an enzyme-linked immunosorbent assay for podophyllotoxin. Int Immunopharmacol 2005, 5:1583-1592.

16. Tothiam C, Phrompittayarat W, Putalun W, Tanaka H, Sakamoto S, Khan IA Ingkaninan K: An enzyme-linked immunosorbant assay using monoclonal antibody against Bacoside $A_{3}$ for determination of jujubogenin glycosides in Bacopa monnieri (L.) Wettst. Phytochem Anal 2011, 22:385-391.

17. Sathe M, Merwyn S, Ghorpade R, Agarwal GS, Rao MK, Rai GP, Kaushik MP. Design and synthesis of immunoconjugates and development of competition inhibition enzyme-linked immunosorbent assay (CIEIA) for the detection of O-isopropyl methylphosphonofluoridate (sarin): an organophosphorous toxicant. J Hazard Mater 2011, 192:1720-1728.

18. Boudet F, Theze J, Zouali M: UV-treated polystyrene microtitre plates for use in an ELISA to measure antibodies against synthetic peptides. J Immunol Methods 1991, 142:73-82.

19. Zouali M, Stollar BD: A rapid ELISA for measurement of antibodies to nucleic acid antigens using UV-treated polystyrene microplates. J Immunol Methods 1986, 90:105-110. 
20. Lagace J, Arsenault S, Cohen EA: Alcian blue-treated polystyrene microtitre plates for use in an ELISA to measure antibodies against synthetic peptides. $\mathrm{J}$ Immunol Methods 1994, 175:131-135.

21. Geerligs HJ, Weijer WJ, Bloemhoff W, Welling GW, Welling-Wester S: The influence of $\mathrm{pH}$ and ionic strength on the coating of peptides of herpes simplex virus type 1 in an enzyme-linked immunosorbent assay. J Immunol Methods 1988, 106:239-244.

22. Güll I, Wirth M, Gabor F: Development of a sensitive and reliable ELISA for quantification of wheat germ agglutinin. J Immunol Methods 2007, 318:20-29.

23. Kim YJ, Cho YA, Lee H-S, Lee YT: Investigation of the effect of hapten heterology on immunoassay sensitivity and development of an enzyme-linked immunosorbent assay for the organophosphorus insecticide fenthion. Anal Chim Acta 2003, 494:29-40.

24. Brun EM, Garcés-García M, Puchades R, Maquieira A: Enzyme-linked immunosorbent assay for the organophosphorus insecticide fenthion. Influence of hapten structure. J Immunol Methods 2004, 295:21-35.

25. Bhandari DR, Shen T, Römpp A, Zorn H, Spengler B: Analysis of cyathanetype diterpenoids from Cyathus striatus and Hericium erinaceus by high-resolution MALDI MS imaging. Anal Bioanal Chem 2014, 406:695-704.

26. Whittington RJ: Evaluation of a simple method for improving the precision of an ELISA detecting antibody in serum. J Immunol Methods 1992, 148:57-64

\section{doi:10.1186/s12896-014-0098-4}

Cite this article as: Shen et al:: Development of an enzyme linked immunosorbent assay for detection of cyathane diterpenoids. BMC Biotechnology 2014 14:98.

\section{Submit your next manuscript to BioMed Central and take full advantage of:}

- Convenient online submission

- Thorough peer review

- No space constraints or color figure charges

- Immediate publication on acceptance

- Inclusion in PubMed, CAS, Scopus and Google Scholar

- Research which is freely available for redistribution 